\title{
New Directions in Traffic Control Analysis through Video Surveillance
}

\author{
R.V.S. Lalitha ${ }^{1, *}$, Divya Lalita Sri Jalligampala ${ }^{2}$, Kayiram Kavitha ${ }^{3}$, Shaik Vahida $^{4}$, Goli Rajasekhar $^{5}$ \\ ${ }^{1,2,4}$ Department of C.S.E, Aditya College of Engineering \& Technology, Surampalem. \\ ${ }^{3}$ Department of C.S.E, Gokaraju Rangaraju Institute of Engineering and Technology, Hyderabad, \\ ${ }^{5}$ Ivy Comptech Pvt 1td., Hyderabad.
}

\begin{abstract}
Traffic management is an increasing problem in both cities and sub urban areas. Authority people involved in traffic management system spend much of time in controlling traffic at junctions. With the advances in technology, monitoring traffic through image processing and video surveillance techniques became the researchers' attention. These techniques help us in controlling traffic as well as to identification of kamikaze drivers and speed violators. The key focus of this research is to do traffic analysis using video surveillance to detect speedy drivers. A wide range of traffic parameters such as flow of traffic, speed of vehicles and vehicle registration number are the major components involved in this research. In this paper, traffic analysis is carried out based on streaming video data with YOLO tool. In this paper an eco system is developed for object detection, vehicle number detection and the speed of the vehicle using computer vision algorithms. With the application tool developed, traffic control authority people can warn the speedy drivers on the fly.
\end{abstract}

Keywords: YOLO tool, Object detection, Video streaming, Classification, ALPR.

\section{Introduction}

Video surveillance acts as a remote eye for traffic authority people. This includes observing vehicles on roads moving with high speed. By viewing selected images at appropriate time helps in reducing accidents and to superimpose safety regulations. Vehicle detection is the most budding topic in urban traffic analysis. In order to reduce accidents, reckless drivers are to be identified. Video surveillance grabbed the attention of researchers to articulate image processing with artificial intelligence capabilities using computer vision [19]. Traffic Control Analysis with Video Surveillance is developed using Image processing techniques in deep learning. Video data is used to analyse the parameters of vehicle like vehicle number, vehicle speed and vehicle categorization. This gives an elaborate way identifying vehicle number, calculating its speed and the type of the vehicle.

\section{Related Work}

F. Mehboob worked on autonomous vehicle detection on roads using videos. Fuzzy logic analysis is used to decide traffic conditions and to take timely actions. For analysis both urban and motorways are considered [1]. W. AL OKAISHI proposed a real time traffic analysis for detection background subtraction method and classification using CNN. The work is carried out using stationary cameras for testing at intersections [2, 16, 17,18]. Fedorov, A addressed the problem of traffic flow estimation using video surveillance. The vehicle count and classification is analyzed using Faster R-CNN by taking 982 video frames with 60,000 objets [3,7]. K. K. Santhosh surveyed about anomaly detection in Intelligent Traffic System. Timely detection of traffic violations helps in reducing accidents. The study includes methods, datasets using computer vision [4, $5,15]$. J, J., R, B proposed novel approach for automatic vehicle management through video surveillance. Object using computer vision algorithms are best suited for this purpose. Automatic vehicle counting is done with blob analysis and background subtraction. Detection of objects is analyzed using boundary block detection algorithms[6]. M. Naphade discussed intelligent video analysis using AI. 5 Tracks are evaluated using promising AI and ML techniques in real world [8-14]. The bibliometric analysis is performed using search key TITLE-ABS-KEY (traffic AND control AND analysis AND using AND video AND surveillance) on 8-82021 from www.scopus.com and the following results are retrieved during 1985 and 2021.

\footnotetext{
* Corresponding author: rvslalitha@gmail.com
} 


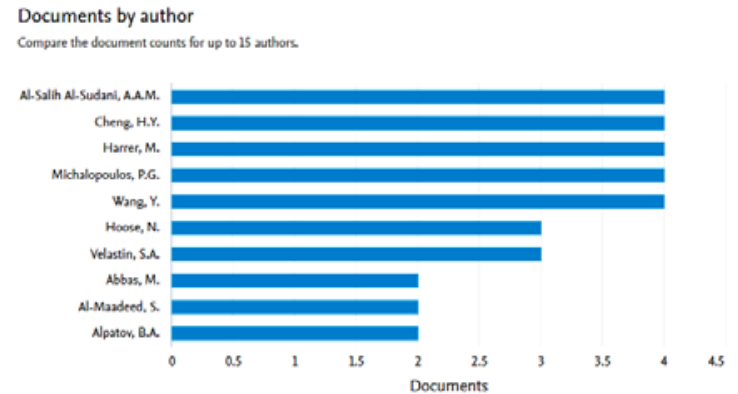

Fig.1(a). Documents by author

The total number of documents retrieved is 364 and the authors mentioned in Fig.1.(i). published 4 documents in this research area. As per the stats Al-Salih Al-Sudani, A.A.M., Cheng, H.Y., Harrer, M. Michalopoulos, P.G. and Wang, Y. published 4 documents and most of the authors published more than 2 documents. The topic is published by several reputed conferences and journals as depicted in Fig.1(b)

Documents per year by source

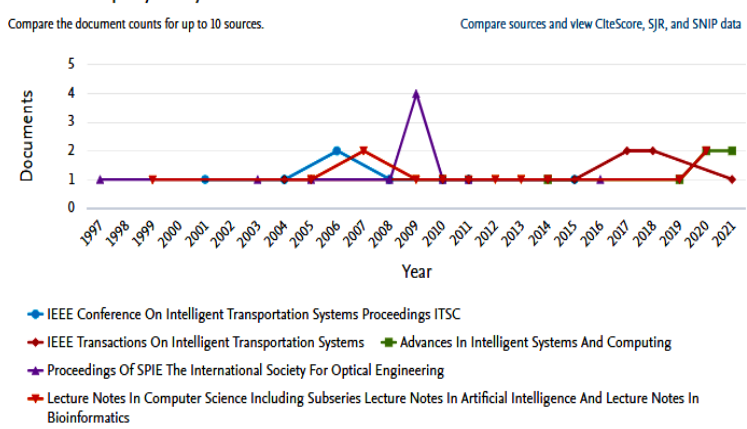

Fig.1(b). Articles published in various reputed journals and conferences.

\section{Traffic analysis through Video Surveillance}

Traffic Control and Analysis System is a dynamic system which analyses the video stream based on the current traffic and also provides traffic rate and classification to clear the traffic in different junctions. By video streaming, the required parameters are extracted using image processing techniques in deep learning. In this system, the detection of vehicles on roads is done using OpenCV and the classification of the vehicles to clear traffic is analyzed using YOLO model. The recognition of License Number is through open ALPR. Finally vehicle speed is detected in real time. The various stages involved are depicted in Fig.2. In this research work, the traffic analysis through video surveillance is mainly performed in three phases: Object detection using YOLO model, Number plate recognition and Computing speed of the vehicle.

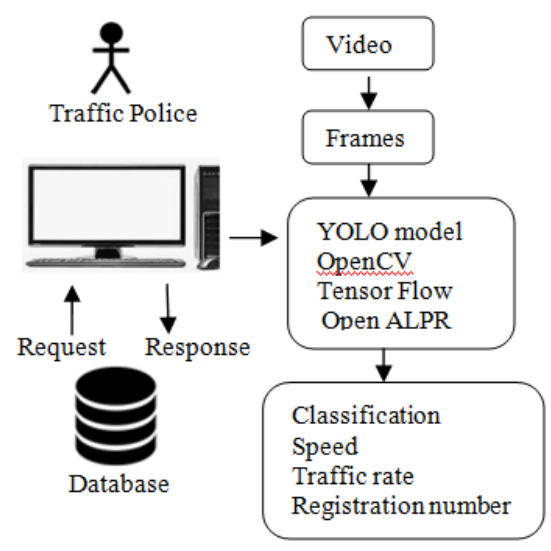

Fig.2. Traffic analysis through Video surveillance

Automatic License Plate Recognition (ALPR) is a technology used for number plate recognition. This is mostly used by Police department at toll gates and road junctions. This uses the principle of Optical Character Recognition on vehicles to detect vehicle registration numbers. Object detection involves recognition of an object in the real world. It includes three stages, recognition, localization and detection. These are very useful for driver assistance based applications.

\section{a) Video capturing}

The video is taken for duration of $1 \mathrm{~min}$ and vehicle detection is performed for a test run. If the camera illusion and light is more the objects will be more correctly identified. The object detection is programmed using OpenCV. The video in mp4 format is submitted to VideoCapture() method. The objects nearby are classified correctly with confidence of $69 \%$ and the vehicle which is far is with with $55 \%$ confidence as shown in Fig.3.

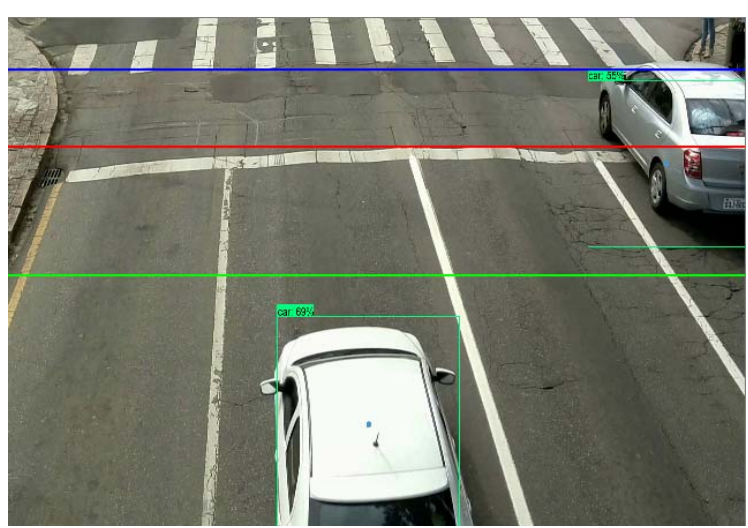

Fig.3. Vehicle Detection in video Stream

b) Vehicle classification

The purpose of this research is to detect the speedy and reckless drivers $[22,23]$. Once the object detection process is completed through video capturing, the vehicle classification takes place to keep track of type of 
vehicles detected. The image database is populated and vehicle classification is performed using YOLOv3 model. The objects are detected from video using detectObjectsFromVideo() method from VideoObjectDetection package. The classification of vehicles and their accuracies are also displayed in Fig.4.

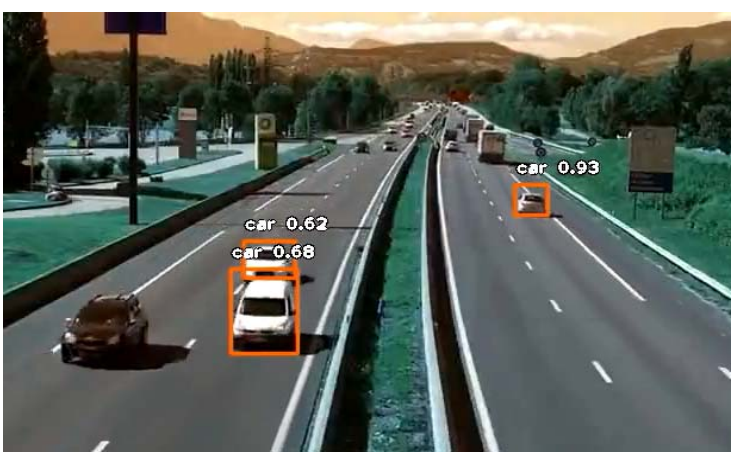

Fig.4. Vehicle Classification in video

c) Traffic rate analysis through video

In this, two components are analyzed. One speed and the other one is number plate detection to warn speed drivers. The speed of the vehicle is calculated based on time of entering into zone and time of leaving. Fig.5. tells about the sequence of actions performed in analyzing traffic count, speed ad number plate detection. Fig.6. exhibit traffic rate analysis using video streaming.

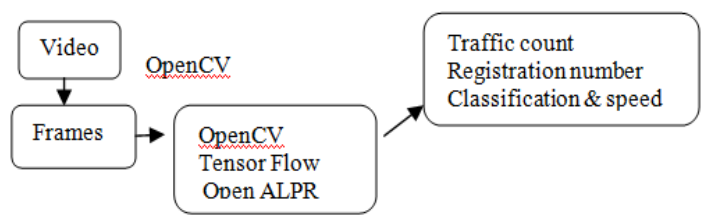

Fig.5. Traffic count, Registration number \& speed

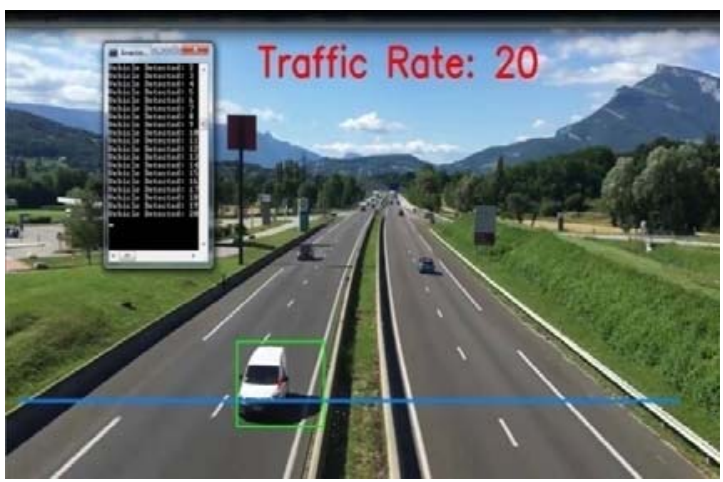

Fig.6. Traffic rate in video

OpenALPR open source library is used to detect number plate detection (Fig.7). The speed and license plate number is displayed.

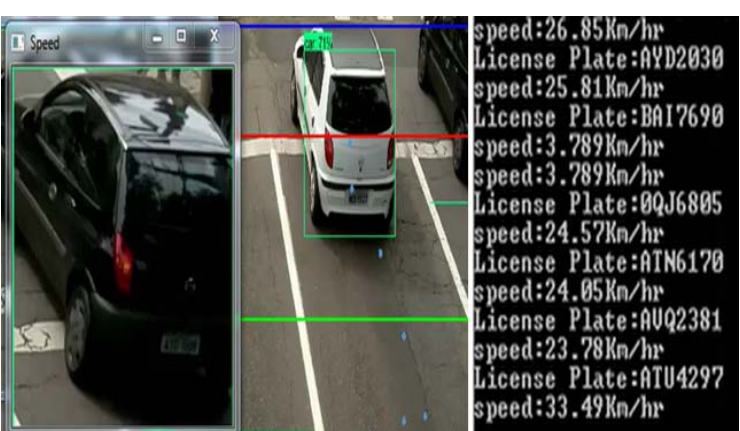

Fig.7. Vehicles speeds and their numbers

d) A dashboard is created using python and MySQL database is used to store traffic parameters. The Authority can make use of this interface to monitor traffic statistics day wise and week wise. If a vehicle is detected with the speed more than the required, then it can be tracked with number plate detected (Fig. 8 and Fig.9). The people of Traffic control authority can access this information through web to keep track of updates.

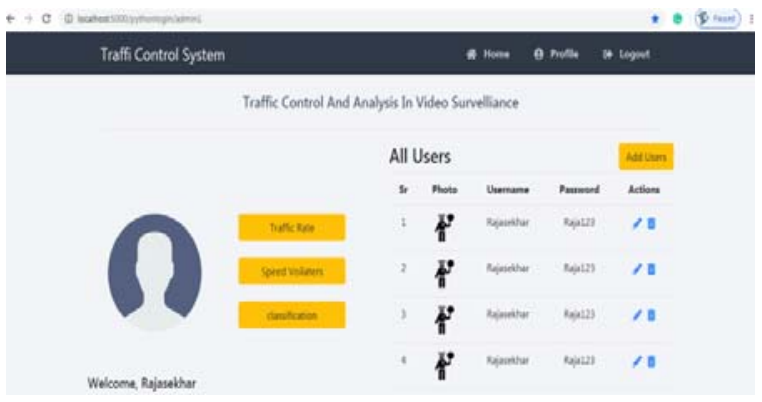

Fig.8. Traffic Analysis detection for speed violators.

The dash board is accessible only to the traffic authority. It requires login credentials to monitor traffic data, speed violators and to classify vehicles on the road. Streaming of video data gives better result than with the magnetic sensors detection. This eco system helps in assessing categorization of vehicle and also the registration information by tracking the details on the number plate.

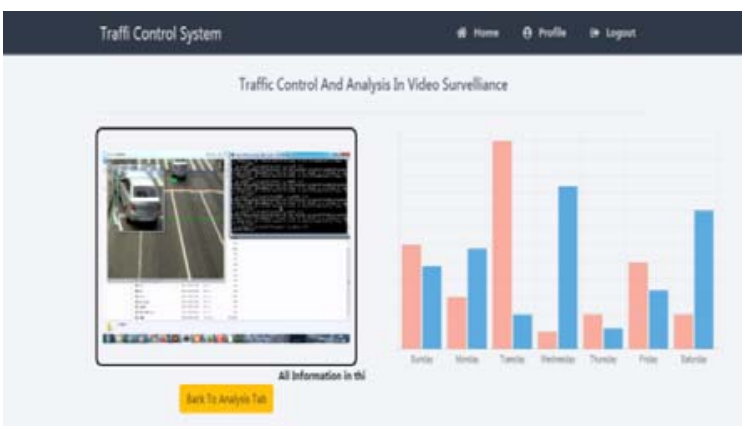

Fig.9. Traffic analysis in a week. 
For visual analytics video surveillance plays key role. The streaming of data from video [24] is a complex method but the conversion of traffic video contents is very much required in detection of objects. The proposed approach outcome gives promising results in conveying emergency information to the speedy drivers. Intelligent monitoring using video surveillance at highways alerts public in maintaining regular speed. Constantly viewing video and observing traffic rate is replaced with the eco system developed in this paper. Web approach [25, 26] drives traffic authority to monitor historic data as well as current day statistics.

\section{Conclusion}

Video surveillance is basically beneficial to the public transportation system. Traffic police can conveniently keep track of traffic updates from the framework developed. The adaptation of intelligent video surveillance at various junctions alerts people in maintaining regular speed of their vehicles. Video surveillance techniques in new directions have exponential growth because of their ability in detection and investigation of number plates. Further extension of this system will also resolve the problem of finding the theft of vehicles. This type of research on video surveillance can serve multiple functions like remote monitoring, tracking, traffic estimation in both public and private sectors.

\section{Acknowledgements}

Authors would like to acknowledge the efforts of K. S.D Shankar, Akshat jain and S.Anusai Reddy in taking videos of traffic data and analyzing them during the progress of this research work.

\section{References}

1. F. Mehboob, M. Abbas and R. Jiang, "Traffic event detection from road surveillance vide os based on fuzzy logic," SAI, 2016, doi: 10.1109/SAI.2016.7555981.

2. W. Al Okaishi, A. Zaarane, I. Slimani, I. Atouf and M. Benrabh, "A Traffic Surveillance System in Real-Time to Detect and Classify Vehicles by Using Convolutional Neural Network," SysCoBIoTS, 2019, pp. 1-5.

3. Fedorov, A., Nikolskaia, K., Ivanov, S. et al. Traffic flow estimation with data from a video surveillance camera. J Big Data 6, 73, 2019.

4. K. K. Santhosh, D. P. Dogra, and P. P. Roy. 2020. Anomaly Detection in Road Traffic Using Visual Surveillance: A Survey. ACM Comput. Surv. 53, 6, Article 119, February 2021.

5. B. Tian, B. T. Morris, M. Tang, Y. Liu, Y. Yao, C. Gou, D. Shen, and S. Tang. 2017. Hierarchical and networked vehicle surveillance in ITS: A survey. IEEE Transactions on Intelligent Transportation Systems 18, 1 (2017), 25--48.

6. J, J., R, B., \& Al-Heety, A. (2021). Moving vehicle detection from video sequences for Traffic Surveillance System. ITEGAM-JETIA, 7(27), 4148 .

7. R. Babitha Lincy, Gayathri, R. Optimally configured convolutional neural network for Tamil Handwritten Character Recognition by improved lion optimization model. Multimedia Tools and Application (2020).

8. M. Naphade, S. Wang, D. C. Anastasiu, Z. Tang, M.- C. Chang, X. Yang, Y. Yao, L. Zheng, P. Chakraborty, A. Sharma, Q. Feng, V. Ablavsky, and S. Sclaroff, "The ${ }^{5 \text { th }}$ ai city challenge," in The IEEE Conference CVPR Workshops, June 2021.

9. Shuai Bai, Zhedong Zheng, Xiaohan Wang, Junyang Lin, Zhu Zhang, Chang Zhou, Hongxia Yang, and $\mathrm{Yi}$ Yang. Connecting language and vision for natural language-based vehicle retrieval. In CVPR Workshop, 2021.

10. Jingyuan Chen, Guanchen Ding, Yuchen Yang, Wenwei Han, Kangmin Xu, Tianyi ao, Zhe Zhang, Wanping Ouyang, Hao Cai, and Zhenzhong Chen. Dual modality vehicle anomaly detection via bidirectional-trajectory tracing. In CVPR Workshop, 2021

11. Keval Doshi and Yasin Yilmaz. An efficient approach for anomaly detection in traffic videos. In CVPR Workshop, 2021.

12. Qi Feng, Vitaly Ablavsky, and Stan Sclaroff. CityFlow-NL: Tracking and retrieval of vehicles at city scaleby natural language descriptions. arXiv:2101.04741, 2021.

13. Marta Fernandez, Paula Moral, Alvaro GarciaMartin, and Jose M. Martinez. Vehicle reidentification based on ensembling deep learning features including a synthetic training dataset, orientation and background features, and camera verification. In CVPR Workshop, 2021.

14. Derek Gloudemans and Daniel B. Work. Fast vehicle turning-movement counting using localization-based tracking. In CVPR Workshop, 2021

15. Luna E, San Miguel JC, Ortego D, Martínez JM. Abandoned Object Detection in Video-Surveillance: Survey and Comparison. Sensors (Basel). 2018;18(12):4290. Published 2018 Dec 5. doi: $10.3390 / \mathrm{s} 18124290$

16. V. Ghanavanth et al., "Smart CCTV surveillance system for intrusion detection with live streaming", 3rd IEEE International Conference RTEICT, 2018.

17. Apoorva Raghunandan, Pakala Raghav and HV Ravish Aradhya, "Object detection algorithms for video surveillance applications", ICCSP, 2018.

18. Archana Kalyankar, Shikha Nema and Umesh Mahind, "Advance and automatic motion detection 
prediction data association with object tracking system", International Conference ICIRCA, 2018.

19. Suma, G.J., Lalitha, R.V.S. Vehicular Ad hoc Networks: A hybrid approach to data dissemination in exigency situations. Wireless Netw 22, 172517372016.

20. Lalitha RVS, Srinivas R, Kumar PSVVSR, Kavitha $\mathrm{K}$,Sameera PVSNS. Intelligent signalling system to control traffic in vehicular ad hoc networks. Indian Journal of Science and Technology. 13(28).

21. Lalitha, R.V.S., Kavitha, K., Krishna Rao, N.V., Rama Mounika, G., Sandhya, V., Smart surveillance with smart doorbell, IJITEE, Volume-8 Issue-8, June 2019,pp 1841-44.

22. Nayak, Padmalaya, K. Kavitha, and Nausheed Khan. "Cluster head selection in wireless sensor network using bio-inspired algorithm." In TENCON 2019-2019 IEEE Region 10 Conference (TENCON), pp. 1690-1696. IEEE, 2019.

23. Reddy, T. Raghunadha, B. Vishnu Vardhan, and P. Vijaypal Reddy. "A survey on authorship profiling techniques." International Journal of Applied Engineering Research 11, no. 5 (2016): 3092-3102.

24. Kumar, Praveen, Ayush Singhal, Sanyam Mehta, and Ankush Mittal. "Real-time moving object detection algorithm on high-resolution videos using GPUs." Journal of Real-Time Image Processing 11, no. 1 (2016): 93-109.

25. Dhanalaxmi, B., G. Apparao Naidu, and K. Anuradha. "Adaptive PSO based association rule mining technique for software defect classification using ANN." Procedia Computer Science 46 (2015): 432-442.

26. Kumar, Singamaneni Kranthi, Pallela Dileep Kumar Reddy, Gajula Ramesh, and Venkata Rao Maddumala. "Image transformation technique using steganography methods using LWT technique." Traitement du Signalvol 36 (2019): 233237. 